\title{
Duration of breastfeeding and gender are associated with methylation of the LEPTIN gene in very young children
}

\author{
Sylvia A. Obermann-Borst', Paul H.C. Eilers², Elmar W. Tobi ${ }^{3}$, Frank H. de Jong ${ }^{4}$, P. Eline Slagboom ${ }^{3,5}$, Bastiaan T. Heijmans ${ }^{3,5}$ \\ and Régine P.M. Steegers-Theunissen ${ }^{1,6,7}$
}

BACKGROUND: Perinatal environmental factors have been associated with the metabolic programming of children and consequent disease risks in later life. Epigenetic modifications that lead to altered gene expression may be involved. Here, we study early life environmental and constitutional factors in association with the DNA methylation of leptin (LEP), a non-imprinted gene implicated in appetite regulation and fat metabolism.

METHODS: We investigated maternal education, breastfeeding, and constitutional factors of the child at $17 \mathrm{mo}$ of age. We measured the DNA methylation of LEP in whole blood and the concentration of leptin in serum.

RESULTS: Duration of breastfeeding was negatively associated with LEP methylation. Low education ( $\leq 12$ y of education) was associated with higher LEP methylation. Boys had higher birth weight and lower LEP methylation than girls. An inverse association was established between birth weight per SD increase $(+584 \mathrm{~g})$ and LEP methylation. High BMI and leptin concentration were associated with lower methylation of $L E P$. CONCLUSION: The early life environment and constitutional factors of the child are associated with epigenetic variations in LEP. Future studies must reveal whether breastfeeding and the associated decrease in LEP methylation is an epigenetic mechanism contributing to the protective effect of breastfeeding against obesity.

P erinatal environmental factors, such as the socioeconomic status (SES) of parents, nutrition, and lifestyle factors are important in the growth, development, and health of a child up to adulthood (1). During pregnancy and early postnatal life, an individual can be programmed for nutritional thrift to adapt and survive in an environment of limited resources and poor nutrition (2). In particular, the mismatch between prenatal and postnatal nutrition is emphasized in association with obesity (3). Epigenetics may be one of the major links between the periconception and perinatal periods in association with health and disease in later life (4). It is described as a biological mechanism to explain gene-environment interactions, in which (heritable) changes in gene expression occur without changes in the DNA sequence (5). DNA methylation is one of the best-understood epigenetic mechanisms and an important programming mechanism of the genome, in which cells and tissues can adapt to past and present environmental exposures (6).

The gene leptin (LEP) has been proposed as an important candidate gene for thrifty phenotypes because it displays epigenetic variation and is involved in the development of obesity and insulin resistance $(4,7,8)$. LEP is primarily expressed in differentiated adipocytes of white fat tissue, and its product, the hormone leptin, has several functions, including the regulation of food intake and the expression of energy-regulating peptides. Defective production of leptin or its receptor is highly associated with obesity (9). Higher leptin levels are associated with adiposity in young children and newborns $(10,11)$. The relationship between the methylation of the LEP promoter and leptin expression has been investigated in vitro during adipose conversion. In human preadipocytes, leptin is not expressed owing to hypermethylation of the LEP promoter. When the preadipocytes mature, the gene is switched on through demethylation (12). Differences in methylation of the LEP promoter influence $L E P$ expression in vitro, which suggests a functional effect of methylation on leptin levels (7).

Illustrative of epigenetic effects on LEP in humans are studies performed on the population exposed to the Dutch famine of 1944-1945. Prenatal exposure to the Dutch famine is associated with an increased methylation of LEP (13). This association is gender-specific and not related to the timing of exposure in pregnancy. The early postnatal period is another critical window in which programming of growth and metabolic functions take place. The injection of leptin during suckling to rodent neonates that are exposed to prenatal under-nutrition prevents them from becoming obese (14). This might be due to decreased methylation of the pro-opiomelanocortin gene and increased expression of the pro-opiomelanocortin-derived peptide alpha-melanocyte stimulating hormone ( $\alpha-\mathrm{MSH})$, which reduces food intake (15). Leptin is also associated with the maturation of the neuroendocrine axis. Neural projection pathways, regulation of food intake,

\footnotetext{
'Department of Obstetrics and Gynecology, Erasmus MC, Rotterdam, The Netherlands; ${ }^{2}$ Department of Biostatistics, Erasmus MC, Rotterdam, The Netherlands; ${ }^{3}$ Department of Molecular Epidemiology, Leiden University Medical Center, Leiden, The Netherlands; ${ }^{4}$ Department of Endocrinology, Erasmus MC, Rotterdam, The Netherlands; ${ }^{5}$ The Netherlands Consortium for Healthy Ageing, Leiden, The Netherlands; ${ }^{6}$ Department of Epidemiology, Erasmus MC, Rotterdam, The Netherlands; ${ }^{7}$ Department of Clinical Genetics, Erasmus MC, Rotterdam, The Netherlands. Correspondence: Régine P.M. Steegers-Theunissen (r.steegers@erasmusmc.nl) 
and energy consumption are disrupted in leptin-deficient mice. Postnatal treatment with leptin, however, restores these projections (16). In children, neuroendocrine appetite regulation is influenced prenatally by leptin derived from the mother and placenta, and synthesized by the fetus, and postnatally by leptin derived from breastfeeding and synthesized by the infant $(17,18)$. Some epidemiological studies have shown that breastfeeding protects against childhood obesity, which seems to be established through the programming of leptin present in breast milk but not in formula $(15,19)$. Reports on the influence of breastfeeding on leptin levels are inconclusive, as both increases and decreases of leptin concentrations have been reported (20).

From this background, we study the methylation of $L E P$ in association with the early environmental and constitutional factors in healthy children at the age of $17 \mathrm{mo}$.

\section{RESULTS}

\section{Characteristics of Mother and Child}

In this study, we included 120 mother and child pairs (Table 1). Maternal smoking was inversely associated with birth weight after adjustment for gender $(\beta=-231 \mathrm{~g} ; P=0.021)$. Boys had a significantly higher birth weight, adjusted for gestational age, than girls. The leptin serum concentrations were slightly higher in girls, albeit not significantly. Of 99 children with available data on breastfeeding, 75 (74.7\%) were breastfed of which 14 were breastfed $<1$ mo. Most of the children were breastfed for at least 1 mo: 21 children were breastfed for $>1-3$ mo $(22 \%), 21$ children were breastfed for $>3-6 \mathrm{mo}(21 \%)$, and 18 children were breastfed for $>6 \mathrm{mo}(18 \%)$. There was no significant association between maternal education and the duration of breastfeeding $\left(\chi^{2}=0.89, P=0.460\right)$. Children who were breastfed for 1-3 mo had a significantly higher serum concentration of leptin (2.8 vs. $2.6 \mathrm{mmol} / \mathrm{l} ; P=0.025)$ than formulafed children. BMI at 17 mo of age was significantly correlated with leptin concentration (Pearson's $r=0.228, P=0.040$ ). Low maternal education ( $\leq 12 \mathrm{y}$ of education) was associated with an increased postnatal growth rate of the child (Pearson's $r=$ $0.234 ; P=0.029$ ).
DNA Methylation of the LEP Gene

Early environmental factors. We measured seven $\mathrm{CpG}$ sites of $L E P$, calculated the methylation per CpG site, and estimated the overall mean methylation (Table 2). In Model 1, low education was associated with a higher $L E P$ methylation (Table 3). Periconception smoking was not associated with overall LEP methylation, but a strong association was revealed for the methylation of one CpG site (CpG 22, $P=0.001)$. Duration of breastfeeding was negatively associated with $L E P$ methylation $(-2.9 \% ; P=0.011)$. In Model 2, the duration of breastfeeding was slightly attenuated but remained significant.

Constitutional factors. To test for possible phenotypic associations with DNA methylation, we analyzed the relationship between gender and birth weight (Table 3). DNA methylation of LEP was $7.3 \%$ lower in boys than in girls (Model 1). In the total group, an inverse association was shown between the birth weight per SD increase $(+584 \mathrm{~g})$ and LEP methylation $(-5.0 \%, P=0.005)$. BMI at 17 mo of age was associated with an overall lower LEP methylation $\left(-3.3 \% / 1.3 \mathrm{~kg} / \mathrm{m}^{2} ; P\right.$ $=0.043$ ). Postnatal growth rate was not associated with $L E P$ methylation. In Model 2, the association between gender and LEP methylation became stronger and the associations with birth weight and BMI at 17 mo of age disappeared.

Leptin serum concentrations. Leptin concentration was significantly associated with the overall methylation of $L E P(-1.7 \%$; $P=0.035$ ) (Table 3 ) and became stronger in Model 2.

Bonferroni correction. After Bonferroni correction, only the association between birth weight and LEP methylation remained significant $(P=0.035)$.

\section{DISCUSSION}

We studied methylation of the LEP gene in children at 17 mo of age in association with early life environmental and constitutional factors because this gene is highly associated with obesity and insulin resistance and is an important candidate for the

Table 1. Maternal and child characteristics

\begin{tabular}{|c|c|c|c|c|}
\hline Characteristics & All children $(n=120)$ & Girls $(n=50)$ & Boys $(n=70)$ & $P$ \\
\hline \multicolumn{5}{|l|}{ Mother } \\
\hline Age at birth (years) & $31.2(27.6-33.6)$ & $31.3(27.1-33.7)$ & $31.2(27.6-33.6)$ & 0.718 \\
\hline Folic acid, periconception & $34(28.3)$ & $14(28.0)$ & $20(28.5)$ & 0.945 \\
\hline Smoking, periconception & $32(26.7)$ & $15(30.0)$ & $17(24.2)$ & 0.485 \\
\hline \multicolumn{5}{|l|}{ Child } \\
\hline Age (months) & $17.0(15.1-18.6)$ & $17.0(15.0-18.3)$ & $17.2(15.5-18.8)$ & 0.409 \\
\hline Leptin, serum (mmol/l) & $2.7(2.4-3.0)$ & $2.8(2.4-3.3)$ & $2.7(2.4-3.0)$ & 0.062 \\
\hline Breastfeeding, $n(\%)^{\mathrm{b}}$ & 74 (74.7) & $32(84.2)$ & $42(68.9)$ & 0.096 \\
\hline Growth rate, $\beta$ & $0.995(0.989-0.998)$ & $0.993(0.984-0.997)$ & $0.996(0.994-0.998)$ & 0.567 \\
\hline $\mathrm{BMI}\left(\mathrm{kg} / \mathrm{m}^{2}\right)$ & $16.6(15.6-17.6)$ & $15.8(15.3-17.2)$ & $17.0(16.2-17.8)$ & 0.002 \\
\hline
\end{tabular}

Data are presented in median (25th-75th percentile) or number (percentage). Data are tested between genders with ANOVA $t$-test or $\chi^{2}$ test.

${ }^{a}$ Adjusted for gestational age. ${ }^{b}$ Defined as any breastfeeding after birth up to the study moment; data available for 99 children. 
thrifty phenotype $(4,7,8)$. We observed significant associations between the methylation of LEP and the duration of breastfeeding, birth weight, BMI, gender, leptin concentration in the child and maternal education. The strongest associations were with gender, leptin concentration, and the duration of breastfeeding.

To our knowledge, this is the first report on the association between the duration of breastfeeding and lower LEP methylation in very young children. Lower methylation of $L E P$ leads to increased expression and higher concentrations of leptin, which is illustrated by our finding of an increased leptin concentration with lower methylation of LEP. This is supported by the functional effect of methylation on leptin levels $(7,12)$. The association between the duration of breastfeeding and LEP methylation is intriguing. In contrast to formula milk, breast milk contains unique growth factors and signaling molecules, as well as leptin. Because of its interaction with almost all neuropeptides that are involved in the regulation of energy balance and food intake, leptin is important

Table 2. Methylation of the $C p G$ sites of $L E P$

\begin{tabular}{lc}
\hline Locus & All children $(n=120)$ \\
\hline CpG 1 & $25.1(4.9)$ \\
CpG 8 & $15.9(5.3)$ \\
CpG 16.17 & $15.6(3.6)$ \\
CpG 19.20 .21 & $13.2(2.9)$ \\
CpG 22 & $45.1(9.2)$ \\
CpG 25 & $43.8(8.5)$ \\
CpG 27 & $6.3(2.4)$ \\
Overall mean (SE) & $23.6(0.3)$ \\
\hline
\end{tabular}

Values are presented as mean (SD) per CpG site. The overall mean (SE) is estimated from the linear mixed model adjusted for the correlation between individual $\mathrm{CpG}$ dinucleotides and bisulfite batch. in the programming of metabolic pathways (17). Therefore, we hypothesize that the breast milk content contributes to programming of the neuroendocrine system by modulating methylation patterns of the LEP promoter. The decrease in LEP methylation could be one of the mechanisms by which breastfeeding contributes to protection against childhood obesity. Although it is well known that breastfeeding is less common in mothers with a low education or low SES, the association between breastfeeding and LEP methylation was not confounded by maternal education (21).

Prenatal exposure to the Dutch famine of 1944-1945 either in the periconception period or late in gestation is associated with an increased methylation of LEP in offspring (13). Therefore, we want to stress that the association we found may not be related only to breastfeeding but could also be mediated through the dietary pattern of the mother during pregnancy, early life feeding practices, and the current dietary pattern of the child. A study in rats showed that animals that were fed a high-fat diet became obese and had a higher methylation of LEP than rats that were fed a normal diet (22). This led to altered metabolic programming of the offspring, resulting in increased body length, decreased insulin sensitivity, and reduced levels of leptin, even in the next generation $(22,23)$. In mice, a low-protein diet during pregnancy and lactation decreased the methylation of the offspring's LEP promoter and led to a stronger induction of leptin secretion in response to a meal (24). Others, however, have reported significant changes in LEP expression either in response to a high-fat diet or weight loss without changes in methylation of the LEP promoter $(25,26)$. Although studying dietary patterns in association with methylation patterns may be difficult, it will be essential to unravel such associations as those described here.

Table 3. Associations between methylation of $\angle E P$ and several variables

\begin{tabular}{|c|c|c|c|c|c|c|}
\hline & \multicolumn{3}{|c|}{ Model 1} & \multicolumn{3}{|c|}{ Model 2} \\
\hline & $\begin{array}{l}\% \text { Absolute } \\
\text { methylation } \\
\text { change }(\mathrm{SE})^{\mathrm{a}}\end{array}$ & $\begin{array}{c}\% \text { Relative } \\
\text { methylation } \\
\text { change (SE) }\end{array}$ & $P^{\mathrm{b}}$ & $\begin{array}{l}\% \text { Absolute } \\
\text { methylation } \\
\text { change }(\mathrm{SE})^{\mathrm{a}}\end{array}$ & $\begin{array}{c}\% \text { Relative } \\
\text { methylation } \\
\text { change (SE) }\end{array}$ & $p^{c}$ \\
\hline Low education & $+2.1(0.8)$ & $+9.1(3.5)$ & 0.008 & $+1.0(0.8)$ & $+4.2(3.4)$ & 0.233 \\
\hline No folic acid, periconception & $+0.1(0.8)$ & $+0.0(0.8)$ & 0.910 & & & - \\
\hline \multicolumn{7}{|l|}{ Constitutional factors } \\
\hline Gender, male & $-1.8(0.7)$ & $-7.3(4.1)$ & 0.010 & $-2.3(0.8)$ & $-9.0(3.9)$ & 0.005 \\
\hline Birth weight ${ }^{e}$ & $-1.2(0.4)$ & $-5.0(1.7)$ & $0.005^{f}$ & $-0.6(0.5)$ & $-2.5(2.1)$ & $0.159^{f}$ \\
\hline Growth rate $e^{e, g}$ & $0.0(0.4)$ & $0.0(0.3)$ & 0.985 & & & - \\
\hline \multicolumn{7}{|l|}{ Biomarker concentration } \\
\hline \multicolumn{7}{|c|}{ 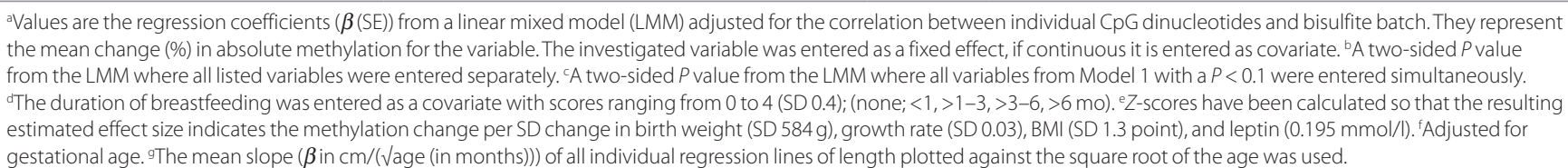 } \\
\hline
\end{tabular}




\section{Articles | obermann-Borstetal.}

Contrary to our expectation, an increase in birth weight and BMI in early childhood was associated with lower LEP methylation. We expected to find higher methylation of LEP because of the association between obesity and hypermethylation that was found in rats (22). Nevertheless, lower methylation of LEP related to an increase in birth weight could explain the strong correlation between an increase in birth weight and leptin levels (20). Because of practical and ethical constraints, we were not able to study the relationship among body composition, different adipose regions, and methylation of LEP. Recent studies also reported on associations between the DNA methylation patterns and constitutional factors in children. Total fat mass and central body fat in children at the age of $9 \mathrm{y}$ have been associated with increased DNA methylation at birth of the retinoid $\mathrm{X}$ receptor gene promoter, a locus involved in insulin sensitivity, adipogenesis, and fat metabolism (27). Furthermore, DNA methylation patterns in cord blood have been associated with body composition at the age of $9 \mathrm{y}(28)$. Although none of these studies looked into $L E P$, these studies do indicate an epigenetic link between DNA methylation at an early age and phenotype in later life. Therefore, our findings may be an early mark and might precede an altered body composition at later age in childhood.

The gender association with LEP methylation replicates an earlier finding (13). Tobi et al. reported a significantly lower LEP methylation in males than in females in a population with a mean age of $57.1 \mathrm{y}$ (SD 5.5). We now show that the gender difference in $L E P$ methylation is already present at a very young age. Gender differences in methylation have been suggested to evolve from sex-steroid expression and $\mathrm{X}$-inactivation resulting in differential methylation of DNA methyltransferases (29). Gender differences are also established in leptin concentration. In general, the concentration is higher in women than in men, and this difference is already present at birth (10). Also in our study population, the leptin concentration is higher in girls than in boys, albeit not significant, probably because of our small sample size. Many underlying explanations for the gender difference in leptin concentration have been presented, such as differences in total fat mass, the distribution of fat mass, involvement of sex-steroid expression, and genetic differences (30). Our results now indicate that epigenetic differences might be involved. One may consider that the higher leptin concentration in girls is in contrast to the higher methylation of LEP. However, our analysis revealed that both in the girls and in the boys an increase in leptin concentration was associated with lower LEP methylation.

The association between low maternal education as proxy for SES and a higher methylation of LEP warrants further investigation. We hypothesize that the adverse effects of the periconception, prenatal, and postnatal environments that are related to low education, such as occupation, nutrition, lifestyle, and health, have left a signature on the methylation of $L E P$. This finding is supported by our recent study showing the same association with INSIGF, which is the overlapping region of insulin-like growth factor 2 (IGF2) and insulin (INS), a locus associated with small-size-for-gestational-age at birth (31). This is in line with a recent exploratory paper showing a distinct genomic methylation pattern associated with early life SES in adult males (32). Although the DNA methylation in their study was also associated with adult SES, there was only little overlap in the genomic region, which suggests an epigenetic variation specifically linked to early SES. Future studies need to identify the strongest factors of low education in association with methylation and to assess whether these changes in methylation have consequences for health and disease. With that knowledge, modifiable risk factors can be identified from which interventions can arise.

In our study, low maternal education was associated with an increased postnatal growth rate of the child; this is in line with the findings in the Generation R study (33). One of the explanations for increased growth rate could be the difference in feeding practices among the socioeconomic groups. However, adjustment for breastfeeding (data not shown) on growth rate did not explain our finding, perhaps because of our small sample size. The increased growth rate could be a response to other adverse intrauterine and postnatal exposures that are related to low maternal education.

We have to address the limitations and strength of our study. A limitation is that we measured DNA methylation in genomic DNA extracted from whole blood and not from other tissues; therefore, we have to be careful in extrapolating our data to tissue-specific methylation. The CpG sites studied in the LEP promoter show similar methylation in peripheral blood and adipocytes in vivo. Furthermore, methylation in adipocytes influences $L E P$ expression in vitro $(7,12)$. However, measurements of $L E P$ mRNA have revealed that especially in children $L E P$ is differentially expressed depending on the adipose region (34).

We measured DNA methylation at the age of $17 \mathrm{mo}$, and it is not known to what extent this reflects methylation patterns set at birth or methylation changes across early postnatal life. Due to the cross-sectional design of the study, we were not able to determine the direction of the associations, i.e., causality or effect, between exposures, phenotypes, and methylation. Furthermore, our sample size together with multiple testing limits the robustness of the inferences that we can make on the associations we found. Therefore, it would be highly informative to study the variation in LEP methylation patterns from birth onwards in a larger group and to examine the relationship with the development of body composition. Although it is often impossible to study the interindividual phenotypic variation that is manifest in inaccessible tissues (such as the brain, visceral fat, and other internal organs and tissues) combined efforts (such as those established through the US National Institutes of Health Roadmap Epigenomics Mapping Consortium) can greatly contribute to this research field (35). Next to studying the overall methylation of LEP, the focus on single CpG sites may be of importance, which is illustrated by our finding of an association between periconception smoking and increased methylation of CpG site 22. The methylation of this site affects the binding and activity of the transcription factor CCAAT/enhancer-binding protein, which modulates expression of LEP (11).

A strength of our study is the standardized study moment of 17 mo after birth, which is a relatively short time after 
pregnancy, thereby minimizing recall bias regarding periconception folic acid use, smoking, and breastfeeding. In conclusion, the present study shows that the variables associated with epigenetic differences in LEP at 17 mo of age are low education, gender, duration of breastfeeding, birth weight, BMI, and leptin concentration. The strongest variables, however, are suggested to be gender, duration of breastfeeding, and leptin concentration. Future studies will be necessary to replicate our findings and to reveal whether they have implications for future health and obesity risk.

\section{METHODS}

\section{Study Sample}

A total of 120 healthy children (boys $n=70$, girls $n=50$ ) at a mean age of $17 \mathrm{mo}$ (SD 2.5) and their mothers were investigated. These motherchild pairs have been previously described in detail (36). In summary, the mothers and their child were recruited from the public child health care centers of "Thuiszorg Nieuwe Waterweg Noord" currently known as "Careyn" in the Rotterdam area (The Netherlands) between October 2003 and January 2007. Public child health care centers are part of the Dutch health care system and are where physicians specialized in child health care regularly check all newborns at standardized times for health, growth, and development. The included children did not have a major congenital malformation or chromosomal abnormality according to the medical records from the regular check up at the child health center up to inclusion at the age of $\sim 17 \mathrm{mo}$. We collected postnatal data on the children and their mothers through self-administered questionnaires sent before the hospital visit, which were checked by a researcher for completeness and consistency at the hospital visit. We extracted and calculated the following data: maternal education, age, periconception folic acid supplement use, smoking, gestational age at birth, child's gender, birth weight, duration of breastfeeding, and BMI at $17 \mathrm{mo}$ of age. We used education level as a proxy for SES and considered it as a cumulative determinant and as the strongest marker of SES (37). We dichotomized this determinant into two categories: low (primary/lower vocational/intermediate secondary education), which corresponds to a maximum of $12 \mathrm{y}$ of ongoing education from the age of 4 , and other (higher secondary/intermediate vocational education or higher vocational/university education). Because periconception folic acid use was previously associated with IGF2 differentially methylated region (DMR) methylation and smoking with INSIGF methylation, we investigated the association of both with LEP methylation $(31,36)$. We included intake of folic acid according to the Dutch recommendation of a daily intake of $400 \mu \mathrm{g}$ of folic acid from at least $4 \mathrm{wk}$ before until $8 \mathrm{wk}$ after conception. Gestational age was based on the first day of the last menstrual period or calculated from the first trimester fetal ultrasound. Data on breastfeeding were derived retrospectively from the mothers in a self-administered home questionnaire. To minimize recall bias, we validated this with the information on breastfeeding from the child's record at the public child health care centers of Careyn. Next to using breastfeeding as a grouping variable, we recoded the different groups into a covariate with scores ranging from 0 to 4 ; (none; $<1,>1-3,>3-6$, $>6 \mathrm{mo}$ ). From the child's record at the public child health care center we collected the child's weight and length, after which his or her BMI was calculated by dividing weight in kilograms by squared height in centimeters. For every child with available growth data from birth onward, we calculated the growth rate by plotting the length against the square root of the age. The slope of each individual regression line $(\beta)$ was used as a variable of the postnatal growth rate. At inclusion, venous blood samples were drawn from all children to measure the serum concentration of leptin. Leptin concentrations were measured in duplicate using a specific Human Leptin Radioimmunoassay kit (Millipore, St Charles, MO). The study protocol was approved by the Central Committee for Human Research in The Hague, The Netherlands, and the Medical Ethical and Institutional Review Board of the Erasmus MC, University Medical Centre in Rotterdam, The Netherlands. All mothers and their respective partners gave their written informed consent on behalf of their participating child.

\section{DNA Methylation Measurements}

DNA methylation of LEP was measured in 120 children. Genomic DNA was isolated from whole blood using the salting-out method (38). One microgram of genomic DNA was bisulfite-treated using the EZ 96-DNA methylation kit (Zymo Research, Irvine, CA) on one of two 96-well plates. Bisulfite-converted DNA-specific PCR primers were used to amplify the investigated region. DNA methylation of $\mathrm{CpG}$ dinucleotides was measured by a mass spectrometry-based method (Epityper; Sequenom, San Diego, CA). The quantitative nature, accuracy, and reproducibility of this method have been shown extensively (39). Details of the measured amplicon, including details of functional relevance, were published previously (40). In short, the amplicon covers the proximal promoter and includes several CpG sites at which the methylation status influences transcription (7). Data quality control and filtering were done as previously described (40). Data filtering consisted of the removal of $\mathrm{CpG}$ dinucleotides for which the measurement success rate was low. Common causes of a lower success rate include fragments bordering on the upper and lower limits of the mass range that can be detected and cases of fragments for which the base of the peak signal in the mass spectrum overlapped another fragment. Details about the primer, the CpG sites included, and biological relevance are provided in Supplementary Table S1 online.

\section{Statistical Analysis}

ANOVA $t$-test and $\chi^{2}$ test were used for the analyses of the characteristics of mother and child between genders. Associations among the study variables were studied using the $\chi^{2}$ test, Pearson's correlation, and linear regression. We applied linear mixed models on the raw data without imputation of missing values to estimate the overall mean methylation, exposure-specific differences, and associations (13). All of the analyses accounted for bisulfite plate and the correlation between the seven $\mathrm{CpG}$ dinucleotides; they were simultaneously entered with the variable under study as fixed effects with overall methylation as the outcome. First, we studied the associations of each of the variables separately with methylation in the linear mixed model. These variables comprised the early environmental factors-maternal low education, no use of folic acid, periconception smoking, and duration of breastfeeding; the constitutional factors of the child-gender, birth weight adjusted for gestational age, growth rate, and BMI; and the biomarker concentration of leptin. These analyses are referred to as Model 1 . Second, we entered all variables with a $P$ value of $<0.01$ for the association with LEP methylation simultaneously in an extensive model referred to as Model 2. Linear mixed model analysis was preferred above the more standard paired $t$-test because it allows analysis of all seven individual CpG dinucleotides together in one test, it accounts for the correlation between adjacent CpG dinucleotides, it includes relevant adjustments within the model on the raw data, and it uses all available data. Absolute changes in DNA methylation are presented as regression coefficient with SE. Absolute changes may seem small but could correspond to a large relative change resulting in functional consequences. Therefore, relative changes in percentage of DNA methylation were calculated by dividing the mean methylation with the risk factor by mean methylation without the risk factor. Because methylation as well as the functional relevance may vary between the individual CpG sites, analyses were also performed for the individual $\mathrm{CpG}$ sites with standard $t$-test and linear regression. These results are provided in Supplementary Table S2 online. Before testing the association of birth weight, BMI, growth rate, and leptin with LEP methylation, $Z$-scores were calculated so that the resulting estimated effect size indicates the methylation change per SD change in birth weight, BMI, growth rate, or leptin. The $Z$-score does not affect any variables other than standardizing the mean to zero and the SD to one and assists in interpreting the results. All $P$ values reported are two-sided. Because multiple statistical tests have been applied, we performed conservative Bonferroni to adjust for multiple comparisons. SPSS 16.0 software (SPSS for Windows; SPSS) was used for all analyses.

\section{SUPPLEMENTARY MATERIAL}

Supplementary material is linked to the online version of the paper at http:// www.nature.com/pr 


\section{ACKNOWLEDGMENTS}

The same children described in this study have been studied in association with methylation of IGF2 DMR, INSIGF, and IGF2R, and the findings have been published in PLOS ONE (PMID 19924280) and the Journal of Developmental Origins of Health and Disease. The research in the current article studies the methylation of leptin with variables that have not been the subject of study in the other publications. We are grateful to the women and children who made this work possible. Furthermore, we thank Bertrand D. van Zelst and Pieter H. Griffioen for laboratory assistance, and the project team of the HAVEN Study for data collection and management. Cissy Siebel is gratefully acknowledged for cooperation with all of the Child Health Care Centers of Careyn. We acknowledge Dennis Kremer for assisting in obtaining and processing DNA methylation data.

\section{STATEMENT OF FINANCIAL SUPPORT}

This work was financially supported by The Netherlands Heart Foundation (grant 2002.B027), the Bo Hjelt Foundation (grant 2005), the European Union-funded Network of Excellence LifeSpan (FP6 036894), and The Netherlands Consortium for Healthy Ageing (NCHA, 05060810) in the framework of The Netherlands Genomics Initiative (NGI)/NWO.

Disclosure: The authors have no conflict of interest to disclose.

\section{REFERENCES}

1. Gluckman PD, Cutfield W, Hofman P, Hanson MA. The fetal, neonatal, and infant environments-the long-term consequences for disease risk. Early Hum Dev 2005;81:51-9.

2. Hales CN, Barker DJ. Type 2 (non-insulin-dependent) diabetes mellitus: the thrifty phenotype hypothesis. Diabetologia 1992;35:595-601.

3. Godfrey KM, Lillycrop KA, Burdge GC, Gluckman PD, Hanson MA. Epigenetic mechanisms and the mismatch concept of the developmental origins of health and disease. Pediatr Res 2007;61(5 Pt 2):5R-10R.

4. Stöger R. The thrifty epigenotype: an acquired and heritable predisposition for obesity and diabetes? Bioessays 2008;30:156-66.

5. Jirtle RL, Skinner MK. Environmental epigenomics and disease susceptibility. Nat Rev Genet 2007;8:253-62.

6. Feng S, Jacobsen SE, Reik W. Epigenetic reprogramming in plant and animal development. Science 2010;330:622-7.

7. Stöger R. In vivo methylation patterns of the leptin promoter in human and mouse. Epigenetics 2006;1:155-62.

8. Noer A, Sørensen AL, Boquest AC, Collas P. Stable CpG hypomethylation of adipogenic promoters in freshly isolated, cultured, and differentiated mesenchymal stem cells from adipose tissue. Mol Biol Cell 2006;17: 3543-56.

9. Rankinen T, Zuberi A, Chagnon YC, et al. The human obesity gene map: the 2005 update. Obesity (Silver Spring) 2006;14:529-644.

10. Hassink SG, Sheslow DV, de Lancey E, Opentanova I, Considine RV, Caro JF. Serum leptin in children with obesity: relationship to gender and development. Pediatrics 1996;98(2 Pt 1):201-3.

11. Ellis KJ, Nicolson M. Leptin levels and body fatness in children: effects of gender, ethnicity, and sexual development. Pediatr Res 1997;42:484-8.

12. Melzner I, Scott V, Dorsch K, et al. Leptin gene expression in human preadipocytes is switched on by maturation-induced demethylation of distinct CpGs in its proximal promoter. J Biol Chem 2002;277:45420-7.

13. Tobi EW, Lumey LH, Talens RP, et al. DNA methylation differences after exposure to prenatal famine are common and timing- and sex-specific. Hum Mol Genet 2009;18:4046-53.

14. Vickers MH, Gluckman PD, Coveny AH, et al. Neonatal leptin treatment reverses developmental programming. Endocrinology 2005;146:4211-6.

15. Palou A, Picó C. Leptin intake during lactation prevents obesity and affects food intake and food preferences in later life. Appetite 2009;52:249-52.

16. Bouret SG, Draper SJ, Simerly RB. Trophic action of leptin on hypothalamic neurons that regulate feeding. Science 2004;304:108-10.

17. Bouret SG. Development of hypothalamic neural networks controlling appetite. Forum Nutr 2010;63:84-93.

18. McMillen IC, Edwards LJ, Duffield J, Muhlhausler BS. Regulation of leptin synthesis and secretion before birth: implications for the early programming of adult obesity. Reproduction 2006;131:415-27.
19. Savino F, Liguori SA. Update on breast milk hormones: leptin, ghrelin and adiponectin. Clin Nutr 2008;27:42-7.

20. Alexe DM, Syridou G, Petridou ET. Determinants of early life leptin levels and later life degenerative outcomes. Clin Med Res 2006;4:326-35.

21. Dubois L, Girard M. Social determinants of initiation, duration and exclusivity of breastfeeding at the population level: the results of the Longitudinal Study of Child Development in Quebec (ELDEQ 1998-2002). Can J Public Health 2003;94:300-5.

22. Milagro FI, Campión J, García-Díaz DF, Goyenechea E, Paternain L, Martínez JA. High fat diet-induced obesity modifies the methylation pattern of leptin promoter in rats. J Physiol Biochem 2009;65:1-9.

23. Dunn GA, Bale TL. Maternal high-fat diet promotes body length increases and insulin insensitivity in second-generation mice. Endocrinology 2009;150:4999-5009.

24. Jousse C, Parry L, Lambert-Langlais S, et al. Perinatal undernutrition affects the methylation and expression of the leptin gene in adults: implication for the understanding of metabolic syndrome. FASEB J 2011;25: 3271-8.

25. Okada Y, Sakaue H, Nagare T, Kasuga M. Diet-induced up-regulation of gene expression in adipocytes without changes in DNA methylation. Kobe J Med Sci 2009;54:E241-9.

26. Marchi M, Lisi S, Curcio M, et al. Human leptin tissue distribution, but not weight loss-dependent change in expression, is associated with methylation of its promoter. Epigenetics 2011;6:1198-206.

27. Godfrey KM, Sheppard A, Gluckman PD, et al. Epigenetic gene promoter methylation at birth is associated with child's later adiposity. Diabetes 2011;60:1528-34.

28. Relton CL, Groom A, St Pourcain B, et al. DNA methylation patterns in cord blood DNA and body size in childhood. PLoS ONE 2012; 7:e31821.

29. El-Maarri O, Becker T, Junen J, et al. Gender specific differences in levels of DNA methylation at selected loci from human total blood: a tendency toward higher methylation levels in males. Hum Genet 2007;122:505-14.

30. Wauters M, Considine RV, Van Gaal LF. Human leptin: from an adipocyte hormone to an endocrine mediator. Eur J Endocrinol 2000;143:293-311.

31. Obermann-Borst SA, Heijmans BT, Eilers PHC, et al. Periconception maternal smoking and low education are associated with methylation of INSIGF in children at the age of 17 months. J Dev Orig Health Dis 2012;3:315-20.

32. Borghol N, Suderman M, McArdle W, et al. Associations with early-life socio-economic position in adult DNA methylation. Int J Epidemiol 2012;41:62-74.

33. Silva LM, van Rossem L, Jansen PW, et al. Children of low socioeconomic status show accelerated linear growth in early childhood; results from the Generation R Study. PLoS ONE 2012;7:e37356.

34. Schoof E, Stuppy A, Harig F, et al. Comparison of leptin gene expression in different adipose tissues in children and adults. Eur J Endocrinol 2004;150:579-84.

35. Heijmans BT, Mill J. Commentary: the seven plagues of epigenetic epidemiology. Int J Epidemiol 2012;41:74-8.

36. Steegers-Theunissen RP, Obermann-Borst SA, Kremer D, et al. Periconceptional maternal folic acid use of $400 \mu \mathrm{g}$ per day is related to increased methylation of the IGF2 gene in the very young child. PLoS ONE 2009;4:e7845.

37. Desai S, Alva S. Maternal education and child health: is there a strong causal relationship? Demography 1998;35:71-81.

38. Miller SA, Dykes DD, Polesky HF. A simple salting out procedure for extracting DNA from human nucleated cells. Nucleic Acids Res 1988;16:1215.

39. Coolen MW, Statham AL, Gardiner-Garden M, Clark SJ. Genomic profiling of $\mathrm{CpG}$ methylation and allelic specificity using quantitative highthroughput mass spectrometry: critical evaluation and improvements. Nucleic Acids Res 2007;35:e119.

40. Talens RP, Boomsma DI, Tobi EW, et al. Variation, patterns, and temporal stability of DNA methylation: considerations for epigenetic epidemiology. FASEB J 2010;24:3135-44. 